Systematic review

\section{Findings from qualitative studies suggest parents of children with disabilities benefit from peer support; whereas findings from quantitative studies are inconclusive}

10.1136/eb-2013-101552

\section{Lutz Goldbeck}

Department of Child and Adolescent Psychiatry/Psychotherapy, University of Ulm Medical Centre, Ulm, Germany

Correspondence to: Dr Lutz Goldbeck, Department of Child and Adolescent Psychiatry/Psychotherapy, University of Ulm Medical Centre, Krankenhausweg 3, Ulm D-89075, Germany;

lutz.goldbeck@uniklinik-ulm.de

Commentary on: Shilling V, Morris C, Thompson-Coon J, et al. Peer support for parents of children with chronic disabling conditions: a systematic review of quantitative and qualitative studies. Dev Med Child Neurol 2013;55:602-9.

\section{Implications for practice and research}

- Qualitative research suggests shared social identity, learning from others, personal growth and supporting others are key ingredients of peer support for parents of children with disabling conditions.

- Evidence for the efficacy of peer support for parents is limited due to the methodical challenges of undertaking controlled studies in this field.

- Future research should aim to identify factors of effective peer support and its utilisation in healthcare settings.

\section{Context}

Healthcare professionals routinely recommend peer support for parents of children with long-term and disabling conditions. Peer support comprises of parent groups and one-to-one parent support in the absence of a healthcare professional or facilitator. Unlike peer support for patients, the effectiveness of peer support for parents has rarely been investigated. Shilling and colleagues undertook a systematic review to identify the perceived benefits of parent peer support to the recipient's health and well-being and family functioning. In addition, studies were evaluated in relation to the type of support offered, duration of support, long-term effects and economic outcomes of peer support.

\section{Methods}

Adopting a non-categorical approach for long-term conditions in childhood, but excluding cancer, Shilling and colleagues systematically searched multiple medical, nursing and allied health databases and grey literature. The methodical quality of the studies was evaluated using criteria described by the UK National Health Service Centre for Reviews and Dissemination and the Cochrane Collaboration. Missing information was requested from the authors of the primary studies where possible. Owing to different types of peer support, heterogeneous outcomes and lack of reporting of statistical details, a meta-analysis of the quantitative studies was not feasible.

\section{Findings}

Seventeen studies were included: nine qualitative studies, seven randomised controlled trials and one mixed-method study. The synthesis of the qualitative studies identified four factors that were perceived as beneficial to individuals offering support and recipients of peer support programmes: 'shared social identity', 'learning from the experience of others', 'personal growth' and 'supporting others'. Lack of shared social identity and a mismatch between perceived need and the peer support available reduced the potential benefit of peer support. Some studies indicated an improvement of a broad range of parents' psychological health outcomes. However, these effects were inconsistent across study findings. No study investigated the effect of peer support on health economic outcomes or healthcare utilisation.

\section{Commentary}

Shilling and colleagues undertook a thorough review of the current literature relating to peer support for parents of children with long-term and disabling conditions. Only a few relevant primary studies were indentified and included in the review; most studies were underpinned by qualitative methods. This paucity of research is noticeable, considering the broad consensus among healthcare professionals to recommend peer support to parent caregivers. The findings of the review suggest peer support has the potential to empower parents and improve their own health, well-being and family functioning. However, findings regarding the effectiveness of peer support were inconsistent across studies. Generalisation from the review findings is difficult, given the heterogeneity of peer support, different outcome measures and the self-selectivity of study participants.

Peer support cannot be investigated using a quasi-experimental design, as support is often opportunistic, for example, parents meet in waiting rooms, in the ward or over the internet. In these circumstances some parents share their experiences, whereas others may remain isolated. Yet, seeking or offering peer support can be considered a way of coping with the child's condition and with the often complex challenges of medical care. Families and parents differ regarding their preferences and opportunities to seek and effectively utilise social support. ${ }^{1}$

As with other coping strategies, peer support cannot be considered as generally effective, because individuals differ regarding their coping styles and preferences. However, peer support can mediate parents' stress response. ${ }^{2}$ Peer support can be a valuable additional resource for parents and can meet their information and support needs through meeting other parents in a similar situation who are willing to share their experiences. The findings of the review indicate that future research should aim to identify factors of effective peer support and its utilisation in healthcare settings. In addition, as the internet becomes increasingly important for seeking health-related information and support, ${ }^{3}$ the effects of internetbased peer communication should be further investigated.

Competing interests None.

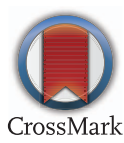

\section{References}

1. Kazak AE. Families of chronically ill children: a systems and social-ecological model of adaptation and challenge. J Consult Clin Psychol 1989;57:25-30.

2. Fidika A, Salewski C, Goldbeck L. Quality of life among parents of children with phenylketonuria (PKU). Health Qual Life Outcomes 2013;11:54.

3. Yli-Uotila T, Rantanen A, Suominen T. Motives of cancer patients for using the internet to seek social support. Eur J Cancer Care (Engl) 2013;22:261-71. 